\title{
ASSOCIATION OF HBSAG WITH SEVERE MALARIA - FACT OR FICTION?
}

Anup K. Das

1. Professor, Department of Medicine \& I/C Hepatology Unit, Assam Medical College, Dibrugarh, Assam.

\section{CORRESPONDING AUTHOR}

Prof. Anup K. Das

Dept of Medicine

Assam Medical College, Dibrugarh, Assam

E-mail: anupkrdas@hotmail.com,

Ph: 00919435702211.

ABSTRACT: India lies in the endemic belt of PF malaria. Annually we are faced with regular outbreaks of severe malaria with its attendent adverse effects on public health in the North-East also. Owing to the diverse geographic, sociopolitical, economic, ethnic and cultural characteristics, this region is also ideal for future "epidemics" of potentially dangerous consequences of HBV infection e.g in Arunachal Pradesh in particular. Epidemiologically, a significant serological association between HbsAg positivity and severe malaria has been reported recently from Africa(,Gambia). But, the multiple factors predisposing to severe malaria, and those influencing the progression of HBV infection have not been elucidated properly. It was postulated that the liver stage parasites are not properly cleared due to concurrent HBV infection of the hepatocytes. Inducible Genetic mutations protective in nature may be a factor. Similarly, those who are vaccinated against HBV are reported to suffer from malaria in South Asia. However, it is premature to draw any definite conclusions as our personal experience give us results to the contrary.

KEYWORDS: Protection from malarial complications, HBsAg, Severe malaria, Malaria \& Hepatitis-B co- infection, Protective genetic mutation

INTRODUCTION: Malaria has been known to occur in India since ancient times ${ }^{1}$. Malaria, especially due to Plasmodium falciparum (PF) remains a leading cause of mortality and morbidity of the human race primarily due to the increasing inability of the insecticides and chemotherapeutic agents we use to eliminate the vectors and the parasite. In addition to cerebral malaria, non cerebral complications like severe anemia, acute renal failure and jaundice are being also increasingly seen and adding to the problems.

Hepatitis B (HBV) infection is an important global public health problem with nearly 400 million carriers. Regions like Soth-East Asia and sub-Saharan Africa fall in high endemic zones for this infection ${ }^{2}$. Similarly, the Indian population is also in the high-risk category. The clinical outcome varies widely ranging from asymptomatic, subclinical, self-limiting disease to endstage liver disease and hepatocellular carcinoma.

The North-Eastern part of India lies in the endemic belt of PF malaria. Annually we are faced with regular outbreaks of severe malaria with its attendent adverse effects on public health in our State. Owing to the diverse geographic,sociopolitical,economic, ethnic and cultural characteristics, this region is also ideal for future "epidemics" of potentially dangerous consequences of HBV infection.The multiple factors predisposing to severe malaria, like the 
ones influencing the progression of HBV infection have not been elucidated properly. However, there have been reports of association of HbsAg carriage with severe malaria ${ }^{3}$.

PATHOGENESIS OF THE SEQUELAES OF SEVERE MALARIA: Although cerebral malaria is still the leading cause of mortality in complicated/severe malaria, still one must be aware of atypical or extracerebral forms ${ }^{4}$ especially the hepatitis and/or renal complications.

Genesis of severe malaria depends upon a complex interplay of the infection and several host factors. There is no absolute or complete infection immunity in malaria 5,6 and the level of parasitemia does not always strictly correlate with the severity of the disease ${ }^{7}$.The most prominent feature in cerebral malaria which is traditionally the commonest severe form encountered in many parts of India, is a dense parasite sequestration in the cerebral capillaries brought about by cytoadherence to endothelial cells and other noninfected RBCs ${ }^{8,9}$. In severe forms other deep organs may be involved by the same process producing a variety of organ dysfunctions.

GENERAL DETERMINANTS OF THE SEVERITY OF MALARIAL INFECTION: Efforts to find out the influencing factors - both genetic and environmental, modifiable and unmodifiable-which can explain some aspects of the variable response to malaria as regards its severity have been going on. In highly endemic areas innate or nonacquired immunity to resist severe malaria are known to exist which depend on genetic constitution of the residents in such regions. Hemoglobinopathies like alpha/beta thalassemia, sickle cell trait, glucose-6-phosphate dehydrogenase deficiency and absence of duffy blood group antigen Fy(a-b-) are some examples $1,10,11,12,13$ suggesting the existence of genetic resistance to malaria.

Because the parasite during its developmental stages presents a large number of antigens to the host immune system, cytokines (e.g. TNF-alpha) and new parasite strains may play a role in triggering severe malaria ${ }^{14,15}$. In addition, PF secrete a number of exoantigens which may induce both protective and potentially harmful immunopathological reaction in humans ${ }^{15}$.

RELATIONSHIP WITH HBSAG WITH SEVERITY OF MALARIAL INFECTION: Epidemiologically, a significant serological association between HbsAg positivity and severe malaria has been reported recently ${ }^{3}$ from Africa (Gambia). It was postulated that the liver stage parasites are not properly cleared due to concurrent HBV infection of the hepatocytes. But in our observation of 303 consecutive complicated malaria cases over a period of 7 years we could not find any HbsAg carrier state in them. According to others also, this association may not be as significant as that found between HbsAg and other diseases like tuberculosis or even rheumatoid arthritis 16,17.

DOES HBV VACCINATION PROTECTS AGAINST SEVERE MALARIA?: If this association is true, then people with HBV vaccination should be at a lesser risk of contracting malaria and its severity later on. But in Africa and Thailand, it was shown that adults and children vaccinated with recombinant HepatitisB vaccine, when compared to unvaccinated controls, had similar rates of PF infection and related morbidity, suggesting that absence of HBV carriage may not prevent the consequences of PF infection 18,19. Vaccines against malaria are at different stages of development and the perfect one still eludes us. A sporozoite specific antigen of PF is the Pfs16. It was found that, when fused with HbsAg to form a protein (Pf16-S) in yeast cells by recombinant method, they formed hybrid particles of a size similar to those present in human 
sera after infection with HBV ${ }^{20}$. It was also observed that, in HBV infection, HbcAg forms an important target for antiviral immunity. Epitope insertion at an immunodominant internal site of $\mathrm{HbcAg}$ reduces the $\mathrm{HbcAg}$ immuno-antigenicity, but it greatly enhances the immunogenicity of the inserted foreign epitopes.This has been used as a carrier moiety to express the circumsporozoite antigen (CS) of PF and form purified hybrid HbcAg-CS proteins. This has been found to protect against experimental PF infection in mouse, possibly by priming specific T-cells 21. Importantly, it was also found that, a pre-existing immunity to $\mathrm{HbcAg}$ did not alter the immunogenicity of this hybrid HbcAg-CS. Similar trials in humans involving hybrid HbsAg-CS plus an adjuvant was also found to protect against PF malaria ${ }^{22}$. These findings would suggest that the association between HbsAg carriage and development of severe malaria is contradictory in the sense that a part of the HBV may in future help us protect against malaria itself. But, it must also be remembered that as in case of most microorganisms, a large number of polymorphic genes and HLA variation may influence the susceptibility or resistance to malaria ${ }^{23}$. Polymorphisms in MHC class I and II loci are of particular interest 24 and it has been reported that the common HLA antigens in Western African population afford protection against severe malaria ${ }^{12}$. In HBV carriage, the reduced level of HLA class I antigen expression on hepatocytes may explain an increased susceptibility to severe malaria. Two HLA-DR13 alleles HLA-DRB1*1301 and*1302 have been known to be associated with resistance to severe malaria and chronic HBV infection 25.

In this backdrop, whether malarial infection induces genetic alterations or mutations to produce or protect against severe malaria in different geographical locations in absence or presence of HbsAg remains to be clarified. We do get a lot of malaria patients annually.Genetic variations in our region needs to be studied in this regard to establish the link(s), if any, of HbsAg carriage with severe malaria because, our genetic constitution may differ from other malarial endemic areas of the world where these links may supposedly exist.

CONCLUSION: As reported from Africa, a study does show an association of the severity of malaria with HbsAg status of the patients. Its premature to draw any definite conclusions as our personal experience give us results to the contrary. We had studied 303 patients with severe Pf malaria in our institute and none were positive for $\mathrm{HbsAg}$.

Susceptibility to malaria is as variable as the disease expression and its severity. Sometimes we encounter extracerebral complications more frequently than the hitherto more common cerebral types in complicated malaria. Many aspects of malarial immunology are still unexplained. Genetic, environmental and other factors which are as yet unknown to us can help us in future to understand this infection and its outcome in different ethnic groups. As the disease is quite common in our part of the country, further works may be taken up to probe the genetic influences on the natural history of both chronic and acute malarial disease.

\section{REFERENCES:}

1. Technical Report Series II Malaria. Report of a Scientific Group of Indian College of Physicians, Bombay.Mishra N.P.(ed).1996. p1, 12

2. Isselbacher K, Braunwald E, Wilson JD et al (Eds): Acute Hepatitis . In Harrison's Principles of Internal Medicine- Companion Handbook. 13 $3^{\text {th }}$ International Edition. McGraw-Hill,Inc. 1994: p525. 
3. Thursz MR, Kwiatkowski D, Torok ME et al: Association of hepatitis B surface antigen carriage with severe malaria in Gambian children. Nat Med April 1995 1(4):374-5.

4. Bag S, Samal GC, Deep N et al: Complicated falciparum malaria. Indian pediatr. Jul1994, 31(7): 821-5.

5. Playfair JHL : Immunity to malaria. Br. Med. Bul. 1982:38(2):153-59

6. Gujar PN, Gujar KN, Parale M: Falciparum malaria. Q. Med. Rev. 1998, 49(3):11-12.

7. Marsh K: Malaria - a neglected disease? Parasitology. 1992,104(Suppl): 53-69.

8. Udeinya IJJA, Schmidt M, Aikawa L et al: Falciparum malaria infected erythrocytes specifically bind to cultured human endothelial cells. Science. 1981, 212:555-57.

9. David PH, Handunnetti SM, Leech JH et al: Rosetting:a new cytoadherence property of malaria infected erythrocytes. Am J Trop Med Hyg. 1988, 38(2):289-97.

10. Allison AC: Protection afforded by sickle-cell trait against subter-tertian malarial infection. Br Med J, 1954 1:290-94.

11. Luzzatto L: Genetics of red cells and susceptibility to malaria. Blood,1979. 54(5):961-76

12. Hill AVS, Allsopp CEM, Kwiatkowski D et al : Common West African HLA antigens are associated with protection from severe malaria. Nature. 1991, 352:595-600.

13. Flint J, Hill AVS, Bowden DK et al: High frequencies of alpha-thalassaemia are the result of natural selection by malaria. Nature, 1986, 321:744-50.

14. Clark IAG, Chaudhri G, Cowden WB : Roles of tumour necrosis factor in the illness and pathology of malaria. Transactions of the Royal Society of Tropical Medicine and Hygiene. 1989, 83:436-40.

15. Jakobsen PH : Plasmodium falciparum malaria parasite exoantigens: their role in disease and in immunity. Dan Med Bull. 1995, 42(1):22-39.

16. Adebajo AO, Smith DJ, Hazleman BL et al: Seoepidemiological associations between tuberculosis, malaria, hepatitis B, and AIDS in West Africa. J Med Virol. Apr 1994, 42(4): 366-68.

17. Adebajo AO, Hazleman BL: The absence of antibodies to malaria and human immunodeficiency virus and the presence of hepatitis B surface antigen as diagnostic markers of rheumatoid arthritis. Clin Rheumatol Dec1993, 12(4) 467-70.

18. Nosten F, Luxemberger C, Kyle DE et al: Randomised double-blind placebo-controlled trial of SPf66 malaria vaccine in children in northwestern Thailand. Shoklo SPf66 Malaria Vaccine Trial Group. Lancet Sept1996, 348(9029):701-07.

19. Sherwood JA, Copeland RS, Taylor KA et al: Plasmodium falciparum circumprotozoite vaccine immunogenicity and efficacy trial with natural challenge quantitation in an area of endemic human malaria in Kenya. Vaccine 1996, 14(8):817-27.

20. Moelans II, Cohen J, Marchand M et al: Induction of Plasmodium falciparum sporozoiteneutralizing antibodies upon vaccination with recombinant Pfs16 vaccinia virus and/or recombinant Pfs16 protein produced in yeast. Mol Biochem Parasitol., 1995, 72(12):179-92.

21. Schodel F, Peterson D, Milich D : Hepatitis B virus core and e antigen: immune recognition and use as a vaccine carrier moiety. Intervirology. 1996, 39(1-2):104-10.

22. Stoute JA, Slaoui M, Heppner DG et al : A preliminary evaluation of a recombinant circumprotozoie protein vaccine against Plasmodium falciparum malaria. RTS-S Malaria Vaccine Evaluation Group. N Eng J Med, 1997, 336(2):86-91. 
23. Hill AV : The immunogenetics of human infectious diseases. Annu Rev Immunol. 1998, 16:593-617.

24. Thursz MR, Thomas HC: Host factors in chronic viral hepatitis. Semin Liv Dis 1997, 17(4):345-50.

25. Davenport MP, Quinn CL, Valsasnini P et al : Analysis of peptide-binding motifs for two disease associated HLA-DR13 alleles using an M13 phage display library. Immunology. 1996, 88(4):482-6. 